\title{
Collateral damage: a case of pylephlebitis in the COVID-19 era
}

\author{
Amram Kupietzky" ${ }^{1 \#}$, Hillel Lehmann ${ }^{2 \#}$, Nurith Hiller ${ }^{3}$, Arie Ariche ${ }^{1}$ \\ ${ }^{1}$ Department of Surgery, Hadassah Medical Organization and Faculty of Medicine, Hebrew University of Jerusalem, Jerusalem, Israel; ${ }^{2}$ Department \\ of Medicine, Hadassah Medical Organization and Faculty of Medicine, Hebrew University of Jerusalem, Jerusalem, Israel; ${ }^{3}$ Department of Radiology, \\ Hadassah Medical Organization and Faculty of Medicine, Hebrew University of Jerusalem, Jerusalem, Israel \\ "These authors contributed equally to this work. \\ Correspondence to: Arie Ariche. HPB Surgery, Hadassah-Hebrew University Medical Center, Mount Scopus, Jerusalem 9765400, Israel. \\ Email: ariche@hadassah.org.il.
}

Submitted Sep 10, 2020. Accepted for publication Jan 11, 2021.

doi: 10.21037/hbsn-20-705

View this article at: http://dx.doi.org/10.21037/hbsn-20-705

Pylephlebitis is an inflamed thrombosis of the portal vein, or of its branches, and is a rare but potentially lethal complication of an intra-abdominal infection. In adults, it is commonly associated with acute appendicitis and diverticulitis. It is often missed due to its non-specific clinical presentation. Usually the diagnosis is done with doppler ultrasonography and computed tomography (CT) scans. Although the major advances in treatments over the past century, this condition is still associated with significant morbidity, with mortality rates remaining as high as $25 \%$ (1). It is important to keep this diagnosis in mind, as part of the differential diagnosis of abdominal pain since it can be treated successfully with early recognition and aggressive intervention.

During the COVID-19 pandemic, a usually healthy 28-year old male presented with generalized malaise, chills, fever, and nausea that had begun 4 days prior to his arrival at the Department of Emergency Medicine. These symptoms where accompanied by 2 episodes of emesis the day prior to his arrival. His review of systems was otherwise unremarkable, and he denied any sick contacts. Vital signs on initial evaluation were a temperature of $38.6^{\circ} \mathrm{C}$, heart rate of $112, \mathrm{BP}$ of $102 / 60 \mathrm{mmHg}$ and a $98 \%$ sat in RA. On physical examination the abdomen was non-distended, there was abdominal guarding with local moderate tenderness on deep palpation of the RLQ, with a positive Aaron and Blumberg sings. mild splenomegaly was note, without other pathological findings. Routine chemistry and hematology revealed white blood cell count of $6.3 \times 10^{3} / \mu \mathrm{L}$ with lymphopenia $0.2 \times 10^{3} / \mu \mathrm{L}$, hemoglobin of $13.8 \mathrm{~g} / \mathrm{dL}$ and platelet count of $87 \times 10^{3} / \mu \mathrm{L}$. Sodium of $133 \mathrm{mmol} / \mathrm{L}$, potassium of $3.3 \mathrm{mmol} / \mathrm{L}$, chloride of $93 \mathrm{mmol} / \mathrm{L}$ and CRP of $13.5 \mathrm{mg} / \mathrm{dL}$, the rest of the laboratory data was within normal limits. A chest X-ray and urine analysis were within normal limits.

The patient underwent a CT of his chest, abdomen and pelvis which showed appendiceal dilatation $(10 \mathrm{~mm})$, wall thickening and enhancement with fat stranding all suggestive of acute appendicitis (Figure 1). On further review of the abdominal CT there was fat stranding through the mesentery reaching the left upper quadrant, with additional filling defects throughout the peripheral portal system veins suggestive of portal vein thrombophlebitis also known as pylephlebitis. The patient was admitted to the surgical ward and prepared for surgery. Intravenous cefuroxime and metronidazole were started in addition to subcutaneous low molecular weight heparin (LMWH). The patient underwent a laparoscopic appendectomy in which a necrotized and inflamed appendix was demonstrated in addition to a severe congested cecal mesentery. Pathological specimen revealed an acute purulent appendicitis with a small perforation. His blood cultures were positive for Escherichia coli, Streptococcus constellatus "milleri" and Bacteroides fragilis susceptible to the chosen empiric antibiotics. The patient was hospitalized for 11 days, his fever persisted for 6 days, his WBC reached a maximum value of $18.4 \times 10^{3} / \mu \mathrm{L}$ and CRP of $23.2 \mathrm{mg} / \mathrm{dL}$. The patient was treated with SC Enoxaparin sodium, levels where monitored using the plasma anti-Xa assays, that indirectly measures the activity of heparins, reaching therapeutic blood concentrations. We performed a Doppler US on POD 12 that demonstrated normal veins flow in the portal and hepatic system including the portal vein. He was discharged after clinical and laboratory parameters 


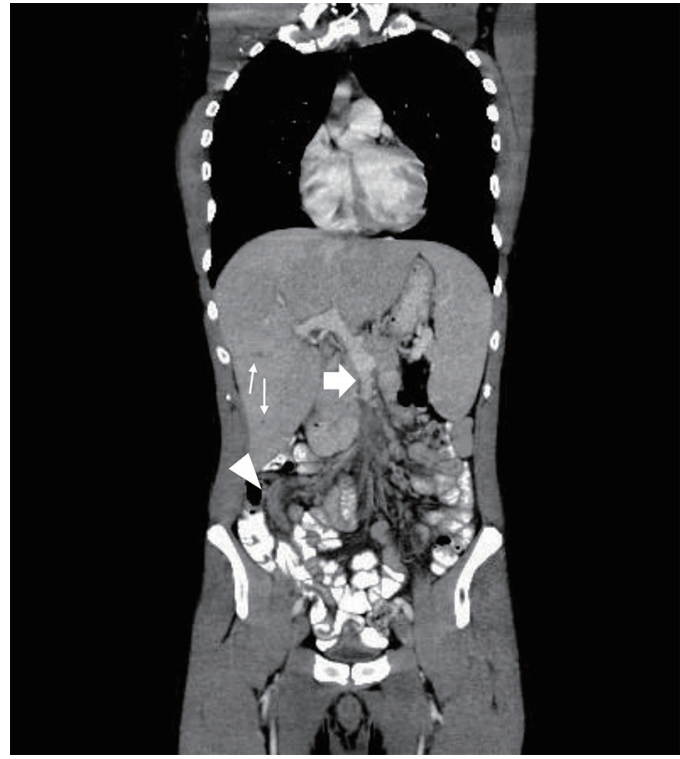

Figure 1 Coronal plane of computed tomography scan at arrival. Arrowhead: the appendix appears swollen with a thick hyperdense wall and with surrounding fat stranding. Small arrows: intrahepatic portal vein branches with no contrast seen within, compatible with portal vein thrombosis. Wide arrow: endoluminal thrombus causing a filling defect in contrast filled Superior mesenteric vein.

improved and completed a 3 weeks regime of cefuroximemetronidazole and LMWH with an outpatient follow-up with our multidisciplinary team.

We present here a case of acute appendicitis complicated with the rare and severe complication of pylephlebitis that occurred during May 2020, the midst of the COVID-19 pandemic in Israel.

It is commonly presumed that Pylephlebitis arises as a polymicrobial intraabdominal infection that drains to the portal venous system (2). The etiologies of these intraabdominal infections include diverticulitis, appendicitis, cholecystitis, pancreatitis, along with less frequent intraabdominal infections, including deep surgical site infections after abdominal surgery (3). Inherited coagulopathies are not associated with a higher incidence of Pylephlebitis, while males have a fairly higher predominance (4).

The clinical presentation of pylephlebitis is usually nonspecific and includes pyrexia, abdominal pain and anorexia (5). Laboratory tests usually demonstrate elevated markers of inflammation, such as leukocytosis, elevated C-reactive protein levels and positive blood cultures.

It is commonly accepted that the treatment must include broad spectrum antibiotics, so that both Gram-negative and anaerobic microorganisms are covered. regarding to the surgical intervention and anticoagulation therapy, different approaches have been described. Surgical intervention can involve urgent appendectomy or a delayed interval appendectomy, without much preference to an open or a laparoscopic approach $(6,7)$. The use of anticoagulation therapy is thought to prevent thrombus propagation, reduce the risk of mesenteric ischemia, improve recanalization and reduces the risk of recurrent thrombosis, however no explicit treatment recommendations have been reported for pylephlebitis (8).

Even with aggressive therapy, the rate of severe complications including liver abscesses, septic shock, mesenteric necrosis, pulmonary emboli and death, is high.

The current COVID-19 pandemic causes fear of exposure in the general public and results in delayed arrival to the emergency department (9). A recent multicenter retrospective analysis comparing the rate of acute appendicitis in 2020 to the corresponding months in 2019 showed a decrease in referrals and incidence but an increase in the rate of severe and complicated appendicitis (10). Our case report demonstrates this phenomenon. As described in other medical conditions, hesitation in seeking medical care results in an increase of morbidity, mortality and higher rate of rare complications. When directly asked, the patient conceded that the COVID-19 pandemic had an impact on his decision to tolerate the fever, chills, and nausea for 4 days before seeking medical attention.

This case calls for increased awareness of complication as pylephlebitis in patients admitted with intra-abdominal infections in the COVID-19 pandemic. As the mortality rates can be higher with complication, early diagnosis and management can reduce mortality in these patients. In this difficult time, surgeons can play a unique role in the re-education of patients to recognize symptoms of lifethreatening surgical conditions and seek appropriate care in a timely fashion.

\section{Acknowledgments}

Funding: None.

\section{Footnote}

Provenance and Peer Review: This article was a standard submission to the journal Hepatobiliary Surgery and Nutrition. The article has undergone external peer review.

Conflicts of Interest: All authors have completed the ICMJE 
uniform disclosure form (available at https://hbsn.amegroups. com/article/view/10.21037/hbsn-20-705/coif). Dr. AA serves as an unpaid editorial board member of Hepatobiliary Surgery and Nutrition. The other authors have no conflicts of interest to declare.

Ethical Statement: The authors are accountable for all aspects of the work in ensuring that questions related to the accuracy or integrity of any part of the work are appropriately investigated and resolved. Written informed consent was obtained from the patient for publication of this manuscript and any accompanying images.

Open Access Statement: This is an Open Access article distributed in accordance with the Creative Commons Attribution-NonCommercial-NoDerivs 4.0 International License (CC BY-NC-ND 4.0), which permits the noncommercial replication and distribution of the article with the strict proviso that no changes or edits are made and the original work is properly cited (including links to both the formal publication through the relevant DOI and the license). See: https://creativecommons.org/licenses/by-nc-nd/4.0/.

\section{References}

1. Plemmons RM, Dooley DP, Longfield RN. Septic thrombophlebitis of the portal vein (pylephlebitis): diagnosis and management in the modern era. Clin Infect Dis 1995;21:1114-20.

Cite this article as: Kupietzky A, Lehmann H, Hiller N, Ariche A. Collateral damage: a case of pylephlebitis in the COVID-19 era. HepatoBiliary Surg Nutr 2021;10(3):418-420. doi: 10.21037/hbsn-2020-705
2. Kasper DL, Sahani D, Misdraji J. Case records of the Massachusetts General Hospital. Case 25-2005. A 40-yearold man with prolonged fever and weight loss. $\mathrm{N}$ Engl J Med 2005;353:713-22.

3. Kanellopoulou T, Alexopoulou A, Theodossiades G, et al. Pylephlebitis: an overview of non-cirrhotic cases and factors related to outcome. Scand J Infect Dis 2010;42:804-11.

4. Choudhry AJ, Baghdadi YM, Amr MA, et al. Pylephlebitis: a Review of 95 Cases. J Gastrointest Surg 2016;20:656-61.

5. Chang YS, Min SY, Joo SH, et al. Septic thrombophlebitis of the porto-mesenteric veins as a complication of acute appendicitis. World J Gastroenterol 2008;14:4580-2.

6. Levin C, Koren A, Miron D, et al. Pylephlebitis due to perforated appendicitis in a teenager. Eur J Pediatr 2009;168:633-5.

7. Stitzenberg KB, Piehl MD, Monahan PE, et al. Interval laparoscopic appendectomy for appendicitis complicated by pylephlebitis. JSLS 2006;10:108-13.

8. Baril N, Wren S, Radin R, et al. The role of anticoagulation in pylephlebitis. Am J Surg 1996;172:44952; discussion 452-3.

9. Lazzerini M, Barbi E, Apicella A, et al. Delayed access or provision of care in Italy resulting from fear of COVID-19. Lancet Child Adolesc Health 2020;4:e10-1.

10. Tankel J, Keinan A, Blich O, et al. The Decreasing Incidence of Acute Appendicitis During COVID-19: A Retrospective Multi-centre Study. World J Surg 2020;44:2458-63. 